\title{
Notfallrisiko: Sommer
}

„Unvorbereitetes Wegeilen bringt unglückliche Wiederkehr.“ J.W. Goethe / Wilhelm Meisters Wanderjahre

$S^{\circ}$ ommer, Sonne, Freizeit und draußen sein - wer möchte das nicht? Aber diesen positiven Begriffen stehen Hitze, Sonnenbrand, Unfälle und Mücken entgegen. Mancher wird erst durch böse Erfahrung schmerzlich daran erinnert, dass auch der Mensch nur gleichberechtigter Teil der Natur ist, vor allem dann, wenn er noch im Zuge des Massentourismus seine ihm bekannte Umwelt verlässt und auch noch unvorbereitet („last minute“) in tropische Regionen reist. Zu selten richtet sich das öffentliche Interesse auf solche Krankheiten.

Fehleinschätzung der eigenen Leistungsfähigkeit und Leichtsinn führen zu Unfällen und Krankheiten, die von der Caisson-Krankheit bis zur Dermatitis solaris reichen. Reiseberatung und Impfprophylaxe, Reiseapotheke und Malariamittel sind keine Kassenleistung mehr, weswegen mancher zusätzliche Kosten scheut und seine Risikounterschätzung (Tauchen mit schwerem Gerät) sich in der Unfallstatistik widerspiegelt. Der Hausarzt muss sich der schwierigen Aufgabe stellen, vorher zu beraten (darf ein Typ I Diabetiker in die Tropen?) und nachher Symptome richtig differentialdiagnostisch einzuordnen (Safari in Afrika und Zeichen eines grippalen Infektes). Aber auch wer zu Hause bleibt und nur im Garten sitzt, hat mit typischen Erkrankungen des Sommers zu kämpfen. In der Hitze verdorbene Nahrungsmittel führen zu Durchfall, und die Unkenntnis der „Verkehrsregeln“ von Bienen und Wespen rächt sich durch Stiche. Allergiker sind im Sommer ohnehin schlechter gestellt als im Winter. Und für die vielen chronisch Kranken gelten erschwerte Bedingungen (phototoxische Nebenwirkungen). Schließlich müssen Herz und Kreislauf den Temperaturausgleich bei großer Hitze leisten, dabei kann bei älteren Menschen die Dauerbehandlung mit Diuretika hinderlich sein. Physiologische Abläufe des Menschen und in die Physiologie eingreifende Medikamente einerseits und die veränderten Umweltbedingungen andererseits machen eine subtile Beurteilung dieser Wechselbeziehungen nötig. Das kann nicht nur bedeuten, dass im Sommer andere Therapiekonzepte erwogen werden müssen, sondern auch, dass mit veränderten Nebenwirkungen und anderen Symptomen zu rechnen ist als in der kalten Jahreszeit. (Haus-) Arzt und Patient müssen im Sommer den veränderten Bedingungen Rechnung tragen.

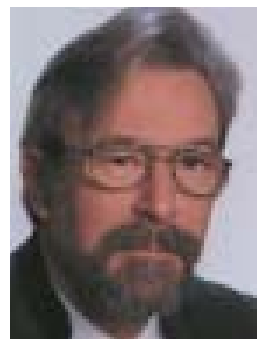

Dr. Ulrich Rendenbach, Duderstadt

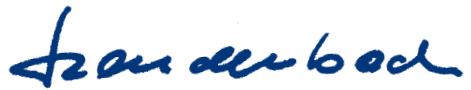

